\title{
Manifestaciones neurológicas en la enfermedad de Kawasaki atípica
}

\author{
Edgar Martínez-Guzmán, ${ }^{1}$ Luisa Berenise Gámez-González, ${ }^{2}$ Francisco Rivas-Larrauri, ${ }^{1}$ \\ Giovanni Sorcia-Ramírez, ${ }^{1}$ Marco Yamazaki-Nakashimada1
}

\begin{abstract}
Background: Kawasaki disease (KD) is a type of systemic vasculitis of unknown etiology. Atypical Kawasaki disease is defined as that where there are signs and symptoms not corresponding to the classical criteria for this nosological entity. Children with atypical Kawasaki disease may present with acute abdominal symptoms, meningeal irritation, pneumonia or renal failure.

Clinical cases: We describe 4 children with ages ranging from 2 to 12 years who had atypical Kawasaki disease, with neurological and gastrointestinal symptoms as part of the systemic presentation of the disease. Treatment consisted of immunoglobulin and corticosteroids with good evolution.

Conclusions: KD is a systemic vasculitis that can involve many territories. Atypical manifestations can mislead the clinician and delay diagnosis. Pediatricians and sub-specialists should be aware of these neurological manifestations in order to provide adequate and opportune treatment.
\end{abstract}

Keywords: Kawasaki disease; Atypical Kawasaki disease; Pancreatitis gallbladder; Hydrops; Seizures; Ataxia

Este artículo debe citarse como: Martínez-Guzmán E, Gámez-González L, Rivas-Larrauri F, Sorcia-Ramírez G, Yamazaki-Nakashimada M. Manifestaciones neurológicas en la enfermedad de Kawasaki atípica. Rev Alerg Mex. 2017;64(3):376-380

${ }^{1}$ Instituto Nacional de Pediatría, Departamento Inmunología.

Ciudad de México, México

${ }^{2}$ Hospital Infantil de Especialidades de Chihuahua, Departamento de Alergia e Inmunología. Chihuahua, Chihuahua, México

\begin{abstract}
Correspondencia: Edgar Martínez-Guzmán dr.edgar.mart78@gmail.com
\end{abstract}

Recibido: 2016-10-07

Aceptado: 2016-12-16 


\begin{abstract}
Resumen
Antecedentes: La enfermedad de Kawasaki es una vasculitis sistémica de etiología desconocida. La modalidad atípica se define como aquella en la cual hay signos y síntomas que no corresponden a los criterios clásicos de esta entidad nosológica. Los niños con enfermedad de Kawasaki atípica pueden presentar síntomas abdominales agudos, irritación meníngea, neumonía o falla renal. Casos clínicos: Describimos 4 niños con edades que oscilaron entre los 2 y 12 años que presentaron enfermedad de Kawasaki atípica, con síntomas neurológicos y gastroinstetinales como parte de la presentación sistémica de la enfermedad. El tratamiento se llevó a cabo con corticosteroides e inmunoglobulina, con los cuales los pacientes evolucionaron satisfactoriamente.

Conclusiones: La enfermedad de Kawasaki es una vasculitis sistémica que puede involucrar numerosos aspectos. Las manifestaciones atípicas pueden confundir al clínico y retrasar el diagnóstico. Los pediatras y subespecialistas deben estar conscientes de estas manifestaciones neurológicas, con el fin de proporcionar tratamiento adecuado y oportuno.
\end{abstract}

Palabras clave: Enfermedad de Kawasaki; Enfermedad de Kawasaki atípica; Pancreatitis; Hidrops vesicular; Convulsiones; Ataxia

\section{Introducción}

La enfermedad de Kawasaki es una vasculitis sistémica de etiología desconocida que afecta principalmente a niños menores de 5 años y representa la principal causa de cardiopatía adquirida en esta población. No se han identificado estudios de laboratorio patognomónicos para el diagnóstico hasta el momento y solo contamos con los criterios clínicos establecidos por el Comité Japonés de Investigación en Enfermedad de Kawasaki, de los cuales se deben cumplir 5 de 6; la Asociación Americana de Corazón establece la fiebre como criterio principal más 4 de los 5 criterios restantes. ${ }^{1}$ Se consideran con enfermedad de Kawasaki atípica los pacientes en quienes hay manifestaciones clínicas no descritas en los criterios diagnósticos principales. ${ }^{1}$

Las formas atípicas se pueden observar en aproximadamente $20 \%$ de los pacientes y representan un factor de riesgo significativo para el desarrollo de enfermedad cardiaca. ${ }^{1}$ El diagnóstico constituye un dilema clínico. Se ha sugerido que estos pacientes pueden tener un peor pronóstico, incluyendo alta tasa de mortalidad: hasta $41 \%$ en los niños con aneurismas de las arterias coronarias. ${ }^{2}$ Los pacientes con una presentación atípica por lo general tienen retraso en el diagnóstico $\mathrm{y}$, consecuentemente, en el tratamiento. ${ }^{1}$
Se ha reportado gran variedad de manifestaciones atípicas ${ }^{1}$ en las cuales están involucrados diversos órganos y sistemas, entre ellos el renal, ocular, gastrointestinal y sistema nervioso central. A continuación describimos 4 pacientes con enfermedad de Kawasaki con manifestaciones neurológicas inusuales.

\section{Caso 1}

Paciente masculino de 7 años de edad que evolucionó durante 15 días con fiebre, dolor abdominal, diarrea y vómito, por lo que fue hospitalizado. Durante la exploración física se encontró hiperemia conjuntival bilateral, labios secos y fisurados; a la palpación abdominal se identificó hepatomegalia. El paciente mostraba deambulación anormal con marcha atáxica. Los exámenes de laboratorio mostraron recuento de leucocitos de $9800 / \mathrm{mm}^{3}$ (5000-14 500) con $90 \%$ de neutrófilos $(51 \%)$, recuento de plaquetas de $144000 / \mathrm{mm}^{3}$ (150 000-350 000), VSG de 45 $\mathrm{mm} /$ hora (4-20 $\mathrm{mm} /$ hora), proteína $\mathrm{C}$ reactiva de $11.8 \mathrm{mg} / \mathrm{L} \mathrm{(0-0.5} \mathrm{mg/L),} \mathrm{aspartato} \mathrm{aminotransferasa}$ de $175 \mathrm{UI} / \mathrm{L}(15-40 \mathrm{U} / \mathrm{L})$, alanino aminotransferasa de $56 \mathrm{UI} / \mathrm{L}(10-40 \mathrm{U} / \mathrm{L})$, bilirrubina total de 1.41 ( $<1.2 \mathrm{mg} / \mathrm{dL}$; directa, $0.26 \mathrm{mg} / \mathrm{dL})$ y albúmina de $2.2 \mathrm{~g} / \mathrm{L}(3.5-5 \mathrm{~g} / \mathrm{dL})$. El análisis del líquido cefa- 
lorraquídeo mostró recuento celular normal y proteinorraquia $(1.3 \mathrm{~g} / \mathrm{L})$. El análisis de la reacción en cadena de la polimerasa para panenterovirus y herpes virus 1 y 2 fue negativo. En la ecografía abdominal se observaron datos de hidrocolecisto.

Se consideró el diagnóstico de enfermedad de Kawasaki incompleto sustentado en la presencia de fiebre, conjuntivitis, queilitis, elevación de transaminasas e hidrocolecisto. Se prescribió una dosis única de la inmunoglobulina intravenosa $(2 \mathrm{~g} / \mathrm{kg})$, aspirina $(5 \mathrm{mg} / \mathrm{kg} / \mathrm{día})$ y metilprednisolona $(15 \mathrm{mg} /$ $\mathrm{kg})$. Durante el tratamiento, la fiebre remitió en un plazo de 48 horas, pero el dolor abdominal persistió, motivo por el cual se realizó ecografía abdominal de control, con la cual se observó distensión de la vesícula biliar con engrosamiento de la pared, perforación de $0.9 \mathrm{~mm}$ en la pared inferior de la vesícula biliar y acumulación de líquido retroperitoneal (Figura 1). La tomografía axial computarizada abdominal mostró bilioma fistuloso y la colangiopancreatografía confirmó hidropesía de la vesícula biliar, perforación y fístula biliar con colección intraabdominal. El paciente fue sometido a colecistectomía y tratado con corticosteroides. La evolución clínica fue satisfactoria, con resolución de la ataxia. No hubo evidencia de anormalidades ecocardiográficas al ingreso o durante el seguimiento.

\section{Caso 2}

Paciente masculino de 12 años de edad previamente sano quien fue hospitalizado por convulsiones tónico-clónicas, fiebre de 4 días de evolución, lesiones papuloeritematosas generalizadas, fatiga, debilidad y vómito. El examen físico reveló hiperemia conjuntival, erupción papuloeritematosa, edema y eritema en palmas y plantas de los pies. Se administró $2 \mathrm{~g} /$ $\mathrm{kg}$ de inmunoglobulina intravenosa y aspirina ante el diagnóstico de enfermedad de Kawasaki. Las pruebas de laboratorio mostraron hemoglobina de $15 \mathrm{mg} / \mathrm{dL}$ (13.5-14.5), leucocitos de $15000 / \mathrm{mm}^{3}$ (4500-13 500), $90 \%$ de neutrófilos (54 \%), $7 \%$ de linfocitos (38\%) y plaquetas de $368000 / \mathrm{mm}^{3}$ (150 000-350 000). El análisis del líquido cefalorraquídeo indicó $85 \mathrm{mg} /$ $\mathrm{dL}$ de proteína $(15-35 \mathrm{mg} / \mathrm{dL}), 95 \mathrm{mg} / \mathrm{dL}$ de glucosa (50-80 mg/dL), 16 células $0-5,101 \mathrm{UI} / \mathrm{L}$ de aspartatoaminotrasferasa $(15-45 \mathrm{U} / \mathrm{L})$ y $216 \mathrm{UI} / \mathrm{L}$ de alaninoaminotransferasa (10-40 U/L). La tomografía axial computarizada cerebral mostró datos de edema y el ecocardiograma fue normal. Con el tratamiento remi- tieron las crisis convulsivas, con adecuada evolución registrada durante las consultas de control.

\section{Caso 3}

Paciente masculino de 2 años previamente sano que por 4 días presentó fiebre, adinamia e hiporexia. Recibió tratamiento antibiótico empírico. Un día después de iniciada la medicación desarrolló erupciones maculares en tronco y extremidades, por lo que se le administró antihistamínicos debido a posible reacción alérgica a fármacos. El paciente fue ingresado después de una crisis convulsiva tónico-clónica generalizada.

A la exploración física de ingreso se identificó irritabilidad generalizada, eritema leve de faringe, adenopatía cervical mayor de $1 \mathrm{~cm}$ y erupción maculopapular que abarcaba la parte superior del pecho y las extremidades. Se sospechó meningoencefalitis viral. Los hallazgos de laboratorio incluyeron hemoglobina de $11.5 \mathrm{~g} / \mathrm{dL}$ (12-12.5), leucocitos de $16070 / \mathrm{mm}^{3}$ (5000-15 500), neutrófilos de $14140 / \mathrm{mm}^{3} 1500-8500$ ), plaquetas de $312000 / \mathrm{mm}^{3}$, VSG de $28 \mathrm{~mm} /$ hora (4-20 mm/hora), PCR de 1:160 $\mathrm{mg} / \mathrm{dL}(0-0.5 \mathrm{mg} / \mathrm{dL})$, aspartato aminotransferasa de $63 \mathrm{UI} / \mathrm{L}(20-60 \mathrm{UI} / \mathrm{L})$, alanino aminotransferasa de $27 \mathrm{UI} / \mathrm{L}$ (10-40 UI/L). El análisis del líquido cefalorraquídeo indicó parámetros dentro de los valores de referencia. En el electroencefalograma no se apreciaron alteraciones. Los cultivos de sangre, líquido cefalorraquídeo y orina fueron negativos para microorganismos patógenos.

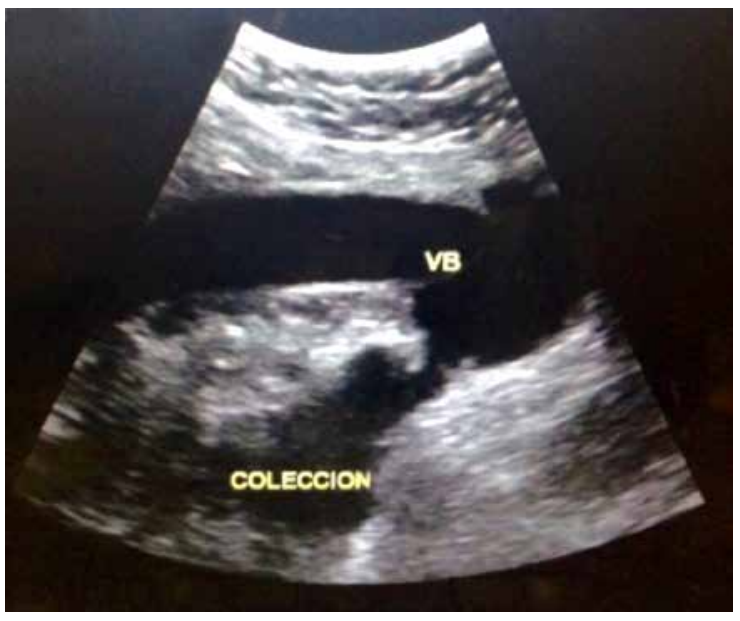

Figura 1. En el ultrasonido se apreció vesícula biliar con salida de líquido (bilis) a la cavidad peritoneal (caso 1). 
Dos días después del ingreso al hospital, el paciente desarrolló hiperemia conjuntival bilateral, lengua en fresa, labios secos y fisurados, edema indurado palmar leve y eritema plantar. El paciente también mostraba eritema perineal. Los hallazgos del examen físico orientaron el diagnóstico a enfermedad de Kawasaki, por lo que se inició la administración de inmunoglobulina intravenosa $(2 \mathrm{~g} / \mathrm{kg})$ y aspirina $(50 \mathrm{mg} / \mathrm{kg} /$ día $)$. El paciente mejoró gradualmente durante las próximas 24 horas. Doce días después del diagnóstico, el paciente presentó descamación fina en los dedos de ambas manos. El ecocardiograma fue normal.

\section{Caso 4}

Paciente masculino de 10 años de edad con historia de parestesias en las extremidades inferiores, convulsiones y habla incoherente. Fue tratado de inicio con fenitoína y diacepam por diagnóstico de epilepsia. Un mes más tarde desarrolló fiebre y lesiones cutáneas en el cuello, cabeza y tronco. Se diagnosticó inicialmente reacción adversa a medicamentos, por lo que se indicó hidrocortisona y difenhidramina. El paciente presentaba fiebre persistente, edema facial, hiperemia conjuntival, queilitis y erupciones generalizadas con descamación. El examen físico reveló eritrodermia generalizada con afectación de la zona perineal, así como descamación de la piel facial, orejas, manos, pies y área periumbilical, con signo de Nikolsky negativo. También se identificó hiperemia conjuntival, queilitis y hepatoesplenomegalia.

Las pruebas de laboratorio indicaron hemoglobina de $11.7 \mathrm{~g} / \mathrm{dL}$ (13.5), leucocitos de $14.400 / \mathrm{mm}^{3}$ (4500-13 500), neutrófilos de $45 \%$ (54\%), linfocitos de $37 \%$ (38\%), plaquetas de $139000 / \mathrm{mm}^{3}$ (150 000-350 000), aspartato aminotransferasa de $3332 \mathrm{UI} / \mathrm{L}(10-60 \mathrm{UI} / \mathrm{L})$ y alanino aminotransferasa de $1114 \mathrm{UI} / \mathrm{L}$ (10-40 UI/L). Mediante la ecografía abdominal se observó colecistitis alitiásica. El ecocardiograma mostró derrame pericárdico. El paciente también desarrolló dolor abdominal; se diagnosticó pancreatitis aguda documentada en ultrasonido abdominal. Recibió inmunoglobulina intravenosa y esteroides, con los cuales mejoró paulatinamente.

\section{Discusión}

El diagnóstico de enfermedad de Kawasaki atípica se basa en manifestaciones clínicas inusuales y no específicas para la enfermedad. Estos datos incluyen síntomas y signos cardiovasculares, gastrointestinales, articulares, urinarios o síntomas neurológicos.

La afección del sistema nervioso central con meningitis aséptica ocurre en $25 \%$ de los pacientes con enfermedad de Kawasaki, pero las convulsiones y la ataxia no son hallazgos frecuentes. Tres de los 4 pacientes presentaron convulsiones y 1 , ataxia. Yun et al. reportaron 6 pacientes $(5 \%)$ con crisis epilépticas entre 121 con enfermedad de Kawasaki, pero ninguno con ataxia. ${ }^{3}$ En un estudio de cohorte de 115 pacientes con enfermedad de Kawasaki, $11(9.5 \%)$ manifestaron ataxia transitoria, como en nuestro caso. ${ }^{3,4} \mathrm{La}$ afectación del sistema nervioso central en forma de parálisis del nervio craneal e infarto cerebral se presenta en menos de $1 \%$ de los pacientes. En estudios post mortem de 30 niños afectados con enfermedad de Kawasaki, Amano et $a l$. encontraron ganglionitis y neuritis de nervio craneal periférico, endoarteritis, periarteritis, coriomeningitis y leptomeningitis, además de atrofia, cambios degenerativos con pérdida de neuronas, gliosis marginal y subependimal, así como degeneración de nódulos gliales alrededor de neuronas. ${ }^{5}$

Mantadakis et al. registraron un paciente diagnosticado con síndrome de hipersensibilidad anticonvulsivante que presentó todos los criterios de enfermedad de Kawasaki, además de alteraciones de las arterias coronarias y derrame pericárdico. ${ }^{6}$ Por su parte, Chinen et al. informaron el caso de un paciente diagnosticado con síndrome de hipersensibilidad anticonvulsivante, por lo que sugirieron el uso de la prueba de la transformación de linfocitos para diferenciar entre los 2 diagnósticos. ${ }^{7}$

En el caso 4 descrito aquí nos inclinamos hacia el diagnóstico de enfermedad de Kawasaki con base en el cumplimiento de los 5 criterios de enfermedad de Kawasaki: afección perineal, vasculitis ocular, vasculitis cutánea, ausencia de eosinofilia, participación de la vesícula biliar y derrame pericárdico, sin embargo, no podemos descartar por completo síndrome de hipersensibilidad anticonvulsivante.

La hidropesía de la vesícula biliar y la disfunción hepática son hallazgos comunes en los niños con enfermedad de Kawasaki, sin embargo, el hidrocolecisto y la perforación de la vesícula biliar son complicaciones extremadamente raras de una vasculitis sistémica. Curiosamente solo existe un caso de hicrocolecisto en el contexto de la púrpura de Henoch-Schönlein: ${ }^{8}$ Hashimoto et al. diagnosti- 
caron una mujer de 75 años de edad con púrpura de Henoch-Schoenlein que fue tratada con corticosteroides e inmunosupresores y presentó perforación de la vesícula biliar con peritonitis biliar. Histopatológicamente solo mostraba células inflamatorias y reacción granulomatosa con hemorragia en el sitio de la perforación. ${ }^{9}$ Colomo Padilla et al. publicaron los hallazgos radiológicos de la vesícula biliar de uno de nuestros pacientes (caso 1). ${ }^{10} \mathrm{Se}$ ha reportado un caso previo de perforación de la vesícula biliar en una niña de 2 años de edad con enfermedad de Kawasaki en quien se utilizó gammagrama con tec- necio-99 y ultrasonido para la detección; la cirugía resolvió la complicación. ${ }^{11}$

La enfermedad de Kawasaki es una vasculitis sistémica con numerosas aristas. Las manifestaciones atípicas pueden confundir al clínico y retrasar el diagnóstico. Los pediatras y subespecialistas deben tener en cuentas las manifestaciones neurológicas para estar en posibilidad de proporcionar un tratamiento adecuado y oportuno.

\section{Agradecimientos}

Los autores agradecen al doctor Alberto Unzueta.

\section{Referencias}

1. Cimaz R, Sundel R. Atypical and incomplete Kawasaki disease. Best Pract Res Clin Rheumatol. 2009;23(5):689-697. DOI: http://dx.doi.org/10.1016/j.berh.2009.08.010

2. Levy M, Koren G. Atypical Kawasaki disease: Analysis of clinical presentation and diagnostic clues. Pediatr Infect Dis J. 1990;9(2):122-126.

3. Yun SH, Yang NR, Park SA. Associated symptoms of Kawasaki disease. Korean Circ J. 2011;41(7):394398. DOI: http://dx.doi.org/10.4070/kcj.2011.41.7.394

4. Alves NR, Magalhães CM, Almeida RF, Santos RC, Gandolfi L, Pratesi R. Prospective study of Kawasaki disease complications: Review of 115 cases. Rev Assoc Med Bras. 2011;57(3):295-300. DOI: http:// dx.doi.org/10.1590/S0104-42302011000300012

5. Amano S, Hazam F. Neural involvement in Kawasaki disease. Acta Pathol Jnp. 1980;30(3):365-373. DOI: http://dx.doi.org/10.1111/j.1440-1827.1980.tb01331.x

6. Mantadakis E, Tsalkidis A, Paraskakis E, Papadopoulou-Legbelou K, Varlamis G, Evangeliou A, et al. Anticonvulsant hypersensitivity síndrome closely mimicking Kawasaki disease. BMJ Case Rep. 2009; DOI: http://dx.doi.org/10.1136/bcr.10.2008.1076

7. Chinen J, Piecuch S. Anticonvulsant hypersensitivity syndrome vs Kawasaki disease: A challenging clinical diagnosis with therapeutic implications. Clin Pediatr (Phila). 2000;39(2):109-111. DOI: http:// dx.doi.org/10.1177/000992280003900206

8. McCrindle BW, Wood RA, Nussbaum AR. Henoch-Schönlein syndrome. Unusual manifestations with hydrops of the gallbladder. Clin Pediatr (Phila). 1988;27(5):254-256. DOI: http://dx.doi. org/10.1177/000992288802700509

9. Hashimoto A, Matsushita R, lizuka N, Kimura M, Matsui T, Tanaka S, et al. Henoch-Schönlein pupura complicated by perforation of the gallbladder. Rheumatol Int. 2009;29(4):441-443. DOI: http://dx.doi. org/10.1007/s00296-008-0727-0

10. Colomo-Padilla AL, Solórzano-Morales SA, Mora-Tizcareño MA, Sorcia-Ramírez G, Garrido-García LM. Perforación de la vesícula biliar asociada con hydrops vesicular como manifestación atípica de la enfermedad de Kawasaki. Informe de un caso. An Radiol Mex. 2015;14(1):123-128.

11. Sty JR, Starshak RJ, Gorenstein L. Gallbladder perforation in a case of Kawasaki disease: Image correlation. J Clin Ultrasound. 1983;11(7):381-384. DOI: http://dx.doi.org/10.1002/jcu.1870110708 